\title{
Analyse de la variabilité spatio-temporelle et modélisation statistique des variations de stock d'eau du sol en forêt
}

\author{
J. CHADOEUF ${ }^{*}$, G. AUSSENAC ${ }^{* *}$, A. GRANIER ** \\ * INRA, Station de Biométrie, Centre de Recherches d'Avignon, \\ Domaine Saint-Paul, F 84140 Montfavet \\ ** INRA, Station de Sylviculture et de Production, \\ Centre de Recherches de Nancy, Champenoux, F 54280 Seichamps
}

\begin{abstract}
Summary
Analysis of temporal and spatial variability and statistical modelling of soil water reserve in forest stands
\end{abstract}

Soil water reserve distribution measured with a neutron moisture meter in forest stands presents a great variability, both in space and in time. An important problem in forest stand water consumption studies, at present based upon water balance methods, is the sampling for soil water content measurement.

An answer to this question is to study the variability of soil water reserve. The authors propose a statistical analysis model allowing the separation of total variation into a time variation and a space variation. The model for interaction separation is based on Principal Component Analysis. Water stock measured at a point $x$ at a time $t$ is decomposed in a general mean to which is added the product of a term linked to time with a term linked to space, and a random term. This expresses the fact that, once the variables are centred, measurements taken at each point of the stand fluctuate proportionally to one another. Random errors are assumed to be independant and to follow a single model. The model paramaters and their variance-covariances are estimated, assuming that the random contribution to variance is small compared to global variance, which has actually proved to be right in the present study.

In a first stage the model parameters are estimated with their statistical characteristics. A strong heterogeneity generally appears between mensurations points, both for the average reserve at each point and for the multiplicative terme linked to space.

In a second stage these parameters are analysed in conjunction with specific characteristics of several different stands. The existence of an important spatial gradient has thus been shown in one of the stands, leading to the elaboration of a map.

It has also been possible to quantify the influence of planting density on water reserve spatial distribution. In the case of a recently thinned stand this analysis lead to study the evolution with time of spatial variations. A strong heterogeneity appears after thinning, followed by a progressive homogenization in conjonction with soil colonization by the roots of remaining trees.

Spatial distribution of the studied parameters in relation to the distance from point of measure to tree has however not been modelled here, due to its very high variability in conjunction with the number of available points of measure in each stand.

Key words: Soil moisture, variability, modelization, spatio-temporal, thinning, water balance. 


\section{Résumé}

La distribution des stocks d'cau mesurés dans des parcelles forestières au moyen d'un humidimètre neutronique présente une variabilité importante, tant dans l'espace que dans le temps. Les études de consommation en eau des peuplements forestiers actuellement basées sur les méthodes de bilan hydrique posent alors un problème important d'échantillonnage des mesures d'humidité du sol. La réponse à cette question passe par une étude de la variabilité des stocks d'eau. Nous proposons un modèle statistique d'analyse, permettant de séparer la variabilité totale en une variabilité temporelle et une variabilité spatiale.

Il s'agit d'un modèle de décomposition de l'interaction, issu des modèles d'Analyse en Composantes Principales. Le stock d'eau mesuré en un lieu $x$ à la date $t$ est décomposé sous la forme d'une moyenne générale à laquelle s'ajoutent le produit d'un terme lié au lieu et d'un terme lié à la date, et une partie aléatoire. Il exprime que, une fois centrées, les mesures en chaque point de la parcelle fluctuent proportionnellement les unes aux autres. Les paramètres du modèle et leurs variances-covariances sont estimés en supposant que la part de variance de la partie aléatoire est faible par rapport à la variance globale, ce qui est vérifié dans notre étude.

Dans une première étape, nous présentons l'estimation des paramètres de ce modèle ainsi que leurs caractéristiques statistiques. De façon générale, il apparaît une très forte hétérogénéité entre points de mesure, tant au niveau du stock moyen en chaque lieu qu'au niveau du terme multiplicatif lié au lieu.

Dans une deuxième étape, ces paramètres sont analysés vis-à-vis des caractéristiques spécifiques des différentes parcelles étudiées. Elle a ainsi permis de dégager l'existence d'un gradient spatial important dans l'une des parcelles et d'en proposer une cartographie.

Elle a également permis de quantifier l'influence de la densité de plantation sur la répartition spatiale des stocks. Dans le cas d'une parcelle récemment éclaircie, cette analyse a permis de suivre l'évolution temporelle des variations spatiales. Une forte hétérogénéité des stocks d'eau apparaît après éclaircie, puis parallèlement à la colonisation du sol par les racines des arbres restants, on assiste à leur progressive réhomogénéisation.

Par contre, la distribution spatiale des paramètres étudiés en fonction de la distance du point de mesure à l'arbre n'a pu être modélisée ici, dans la mesure où elle présentait une variabilité trop importante par rapport au nombre de points de mesure disponibles dans chaque parcelle.

Mots clés : Humidité du sol, variabilité, modélisation, spatio-temporel, éclaircie, bilan hydrique.

\section{Introduction}

L'évaluation de l'évapotranspiration est un élément essentiel pour la compréhension du fonctionnement des écosystèmes forestiers, notamment en ce qui concerne leur production. La mesure de l'évapotranspiration des forêts est rendue difficile de par l'hétérogénéité des peuplements, tant au niveau du sol que de la structure du couvert.

Les différentes approches développées actuellement pour estimer l'évapotranspiration en forêt se classent en trois groupes :

- mesure des flux aériens, au-dessus des houppiers, avec le développement récent de la méthode des fluctuations. Ces méthodes supposent des parcelles homogènes et de grande extension horizontale, et ne permettent pas d'explorer la variabilité spatiale des peuplements ; 
- mesure des flux liquides dans le tronc des arbres, qui permet d'estimer la transpiration, mais pas l'ETR totale ;

- méthode du bilan hydrique, par la mesure des variations d'humidité du sol. Cette méthode a connu un développement important grâce à l'utilisation des humidimètres neutroniques.

L'étude de la variabilité spatiale de la réserve hydrique dans le sol et de son évolution dans le temps a fait l'objet d'un certain nombre de travaux; citons ceux de Nielsen et al. (1973), Vauclin (1983), Rambal et al. (1984), Chadoeuf (1984), Milly \& EAGLESON (1987), réalisés aussi bien sur des sites agricoles que forestiers. Cette variabilité est le plus souvent mise en relation avec celle des caractéristiques hydrodynamiques des sols ; dans le cas des peuplements forestiers peut aussi s'y ajouter l'effet des arbres.

Plusieurs séries d'expérimentations menées en Lorraine ont permis de comparer les différents termes du bilan hydrique en fonction de l'essence, de la structure du peuplement et des interventions sylvicoles (Aussenac \& Granier, 1979; Aussenac \& Boulangeat, 1980; Aussenac et al., 1982). Elles ont aussi mis en évidence la variabilité des réserves en eau du sol et l'objet de ce travail est précisément d'utiliser le nombre important de mesures ayant servi de base à ces travaux pour :

- caractériser la variabilité spatio-temporelle des variations d'humidité du sol,

- en proposer une modélisation de type statistique.

\section{Matériel et méthodes}

\subsection{Les données expérimentales}

Le tableau 1 résume les principales caractéristiques des trois peuplements ayant servi de base à cette étude ; ils sont situés en forêt domaniale d'Amance, à $15 \mathrm{~km}$ à l'est de Nancy (altitude $250 \mathrm{~m}$, latitude $48^{\circ} 44^{\prime} \mathrm{N}$, longitude $6^{\circ} 14^{\prime} \mathrm{E}$ ). Les précipitations annuelles sont voisines de $700 \mathrm{~mm}$, la température moyenne annuelle est de $9,1^{\circ} \mathrm{C}$. Le sol est de type sol brun faiblement lessivé à pseudogley; l'horizon d'engorgement apparaît vers 60 à $80 \mathrm{~cm}$ de profondeur.

L'humidité du sol a été mesurée avec un humidimètre gammaneutronique N.E.A. ; les tubes d'accès (voir tabl. 1) ont une longueur utile de 150 à $220 \mathrm{~cm}$.

La fréquence des mesures en phase estivale est en général de 7 jours. Les cumuls de lame d'eau ont été calculés sur les 150 premiers centimètres de profondeur de sol.

Dans un premier temps, nous analyserons la parcelle feuillue, qui a fait l'objet des mesures les plus nombreuses. Pour cela, nous commencerons par présenter les résultats les plus significatifs de l'analyse de données, réalisée essentiellement au travers d'analyses en composantes principales, avant de choisir un modèle plus spécifique à l'expérience.

Nous disposons de 23 points dont 20 sont répartis sur une grille. Une estimation du variogramme et sa modélisation était de ce fait délicate. Par contre, nous disposons 


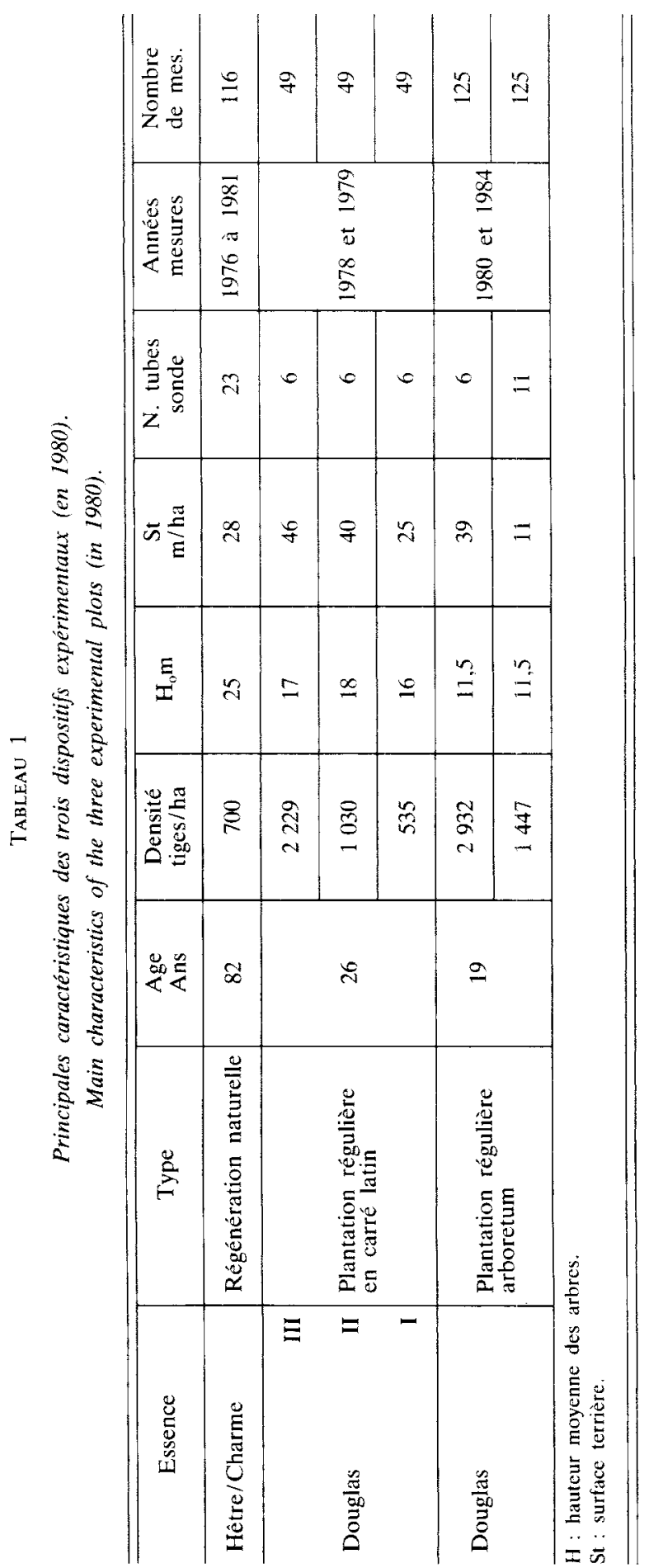


d'un relativement grand nombre de mesures dans le temps (116 dates), et nous chercherons à en tirer parti par une modélisation dont nous présentons les grandes lignes en 2.2 .

Ensuite, nous appliquerons ce modèle aux deux peuplements résineux :

- l'un présentant trois densités de plantation,

- l'autre ayant été soumis à une éclaircie en 1980.

Dans cette parcelle, au cours de l'été 1983, une partie du sol a été recouverte d'une bâche en matière plastique pour étudier l'influence de la suppression des précipitations sur le bilan hydrique.

\subsection{Le modèle d'interaction}

Le modèle utilisé est le suivant :

$y(i, j)=a(i)+t . c(i) \cdot b(j)+e(i, j)$

où $1<\mathrm{i}<\mathrm{I}$ représente les tubes $(\mathrm{I}=23$ dans la parcelle feuillue $)$

$1<\mathrm{j}<\mathrm{J}$ représente les différentes dates de mesure

$\mathrm{y}(\mathrm{i}, \mathrm{j}) \quad$ stock d'eau au point $\mathrm{i}$ à la date $\mathrm{j}$

avec les contraintes $\Sigma b(j)=0$ et $\Sigma c^{2}(i)=1$

$$
\Sigma b^{2}(j)=1
$$

les e (i,j) étant des variables aléatoires indépendantes et identiquement distribuées.

C'est en fait un modèle simple de structuration de l'interaction. Il revient à ne conserver que le premier axe de l'Analyse en Composantes Principales. Il traduit la proportionnalité des variations de stock d'eau des tubes aux différents points de la narcelle : entre le point il et $\mathrm{i} 2$, le coefficient de proportionnalité est de c (i1)/c (i2). Si par exemple, toutes les réserves en eau des tubes fluctuaient de la même façon, ce rapport serait de 1 , dont $\mathrm{c}(\mathrm{i})=1 / \sqrt{\mathrm{I}}$, I étant le nombre de tubes.

Ce modèle permet de séparer l'effet date, représenté par les paramètres b $(j)$, des effets spatiaux que l'on retrouve dans les paramètres a (i) et c (i).

Ainsi que cela a déjà été précisé auparavant, nous nous intéresserons surtout aux paramètres spatiaux dans la mesure où ils conditionnent toute estimation à l'échelle de la parcelle, tant en ce qui concerne les valeurs du stock d'eau à une date donnée que les variations entre deux dates.

Si on suppose que e $=$ al.f, où al est un réel tendant vers 0 , les f étant des erreurs à support borné, on peut montrer que les paramètres convergent en norme $\mathrm{L}^{2}$ quand al tend vers 0 . On a en particulier pour les paramètres a (i) et $b(j)$ qui nous intéressent plus particulièrement, en notant â l'estimateur du paramètre a et $\alpha(i,$.$) la moyenne$ des $\alpha(\mathbf{i}, \mathbf{j})$ :

- $\hat{a}(i)=y(i,$.$) et, quand f$ tend vers 0 ,

$\hat{a}(i)=a(i)+e(i,$.$) donc, en particulier,$

- $E(\hat{a}(i))=a(i)$ : il n'est pas biaisé

- $\operatorname{cov}(\hat{a}(\mathrm{i} 1), \hat{a}(\mathrm{i} 2))=\sigma^{2} / \mathrm{J}$ si i1 $=\mathrm{i} 2$

0 sinon 
- c (i) : ce sont les composantes du premier vecteur propre de la matrice de variance. On obtient quand $f$ tend vers 0 :

$$
\begin{aligned}
& \hat{\mathrm{C}}=\mathrm{C}+\left(|\mathrm{I}|-\mathrm{C}^{\prime}\right) . \mathrm{E} . \mathrm{B} / \mathrm{J} \\
& \text { où } \mathrm{C} \text { est le vecteur des } \mathrm{c}(\mathrm{i}), \mathrm{C}^{\prime} \text { son transposé } \\
& |\mathrm{I}| \text { la matrice unité, } \\
& \mathrm{E} \text { la matrice des }(\mathrm{e}(\mathrm{i}, \mathrm{j})-\mathrm{e}(\mathrm{i}, .)) \\
& \mathrm{B} \text { le vecteur des } \mathrm{b}(\mathrm{j})
\end{aligned}
$$

d'où $\mathrm{E}(\hat{\mathrm{C}})=\mathrm{C}$$$
\operatorname{Var}(\hat{\mathrm{C}})=\left(|\mathrm{I}|-\mathrm{CC}^{\prime}\right) \boldsymbol{\sigma}^{2} / \mathrm{J}^{2}
$$$$
\text { et en particulier } \operatorname{Var}(\hat{C}(i))=\left(1-C^{2}(i)\right) \sigma^{2} / t^{2}
$$

- la somme des carrés résiduels vérifie de même au $2^{\mathrm{c}}$ ordre $\mathrm{E}(\mathrm{SCR})=$ $(\mathrm{I}-1)(\mathrm{J}-2) \sigma^{2}$ ce qui nous permettra d'estimer la variance.

\section{Résultats}

\subsection{Cas de la parcelle feuillue}

\subsection{Analyse des données}

Rappelons que nous effectuons une analyse sur le tableau des lames d'eau, les dates sont prises comme individus, et les tubes comme variables. Nous avons donc un tableau $23 \times 116$. tabl. 2) :

On note une assez grande variabilité des réserves d'eau entre les tubes (voir

- les moyennes fluctuent de $470 \mathrm{~mm}$ à $534 \mathrm{~mm}$,

- les écarts-types vont de $21 \mathrm{~mm}$ à $58 \mathrm{~mm}$ soit des variations allant du simple à plus du double.

Toutefois, les corrélations entre tubes sont très fortes, les plus faibles étant de 0,7 (les plus fortes sont de plus de 0,9 ), ce qui indique déjà un comportement d'ensemble des tubes.

Nous avons ensuite procédé à une analyse en composantes principales.

Nos données étant homogènes, nous avons préféré effectuer cette analyse sur la matrice de variance. Elle nous montre un premier axe très important. Nous obtenons en effet les pourcentages d'inertie de :

$$
92,3-3,5-1,5-0,6-0,5
$$

Ce premier axe de très forte inertie est un effet «taille ». Il signifie tout simplement que les réserves en eau sont toutes globalement fortes ou faibles en même temps. Cet axe décrit donc l'état hydrique moyen de la parcelle. Mais au-delà de cette évidence, il faut remarquer que l'amplitude des fluctuations est très différente d'un tube à l'autre (effet bien représenté par les composantes du premier vecteur propre) et que les 92 p. 100 d'inertie montrent que les variations d'un tube sont quasiment proportionnelles d'un tube à l'autre. 
Tableau 2

Variabilité de la réserve en eau du sol (sur $1.5 \mathrm{~m}$ de profondeur) mesurée dans la parcelle feuillue (moyennes sur 116 dates).

Soil water variability (up to $1.5 \mathrm{~m}$ depth) in the beech stand (means of 116 dates).

\begin{tabular}{c|c|c}
\hline \hline Tube & $\begin{array}{c}\text { Moyenne } \\
(\mathrm{mm})\end{array}$ & $\begin{array}{c}\text { Ecart-type } \\
(\mathrm{mm})\end{array}$ \\
\hline 1 & 527 & 20,7 \\
2 & 503 & 27,9 \\
3 & 476 & 41,8 \\
4 & 481 & 50,1 \\
5 & 489 & 34,7 \\
6 & 512 & 30,9 \\
7 & 534 & 26,1 \\
8 & 524 & 24,8 \\
9 & 505 & 32,1 \\
10 & 489 & 39,9 \\
11 & 460 & 58,2 \\
12 & 492 & 38,0 \\
13 & 475 & 48,5 \\
14 & 505 & 32,5 \\
15 & 501 & 31,2 \\
16 & 506 & 40,4 \\
17 & 492 & 42,1 \\
18 & 496 & 38,8 \\
19 & 488 & 41,1 \\
20 & 522 & 31,5 \\
21 & 514 & 31,2 \\
22 & 470 & 44,6 \\
23 & 508 & 35,2 \\
\hline \hline
\end{tabular}

Le deuxième axe montre justement un premier écart à cette proportionnalité différenciant les stocks «moyens » (desséchements moyens dans les périodes normales) des stocks très faibles correspondant à la sécheresse de 1976 (correspondant aux onze dates s'étalant entre le 30/06/76 et 29/09/76) et, à un degré moindre, de 1979 (le 03/ 08/79 et le 09/09/79). Elle correspond à un ralentissement progressif de la baisse des stocks d'eau de plus faible amplitude quand la sécheresse s'accentue (on notera que les deux épisodes orageux intervenus en 1976 durant cette période n'ont pas annulé la situation de sécheresse). Si ces deux vecteurs propres sont donc très liés, ils correspondent à deux phénomènes très différents.

\section{Analyse des vitesses de variation}

Une deuxième analyse a porté sur les vitesses de variation :

$v(i, j)=(y(i, j+1)-y(i, j)) /(n(i, j+1)-n(i, j))$

où $n(i, j)$ est la date de la $1^{\text {re }}$ mesure du tube $i$. 
Une analyse sur la matrice empirique des moments d'ordre 2 a été effectuée. On obtient alors les pourcentages d'inertie suivants :

$$
81,5-3,5-2,5-2-1,6
$$

Le premier axe décrit comme précédemment la proportionnalité des variations de stock d'eau d'un tube à l'autre. Par contre, cette analyse atténue fortement l'effet sécheresse mis en évidence précédemment. De façon générale, on retrouve sur les points excentrés des écarts à la proportionnalité dus :

- soit à une remontée de stock à partir d'un niveau faible,

- soit à une remontée alors que le niveau moyen est déjà haut.

On retrouve donc bien un effet sécheresse dans le premier cas, alors que le second met plutôt en évidence des effets vraisemblablement dus à un ruissellement alors que le sol est saturé.

\section{Conclusions}

Nous retiendrons de cette analyse la proportionnalité des variations des stocks d'eau aux différents points de mesure. Elle est mise en défaut lors des dessèchements importants, et à un degré moindre lors des remontées de stock alors que le niveau est déjà haut. Nous écarterons donc de cette étude les données de l'année 1976 (22 dates).

Cela revient alors à choisir comme modèle d'évolution des stocks le modèle présenté au 2.2 .

\subsection{Le modele d'interaction}

\section{a) Estimation}

Après suppression des données liées aux dates précédentes, on obtient un tableau des stocks d'eau de 23 variables (les tubes) et 94 individus (les dates).

La somme de carrés résiduels atteint 1340000 , ce qui nous donne une variance estimée de $66 \mathrm{~mm}^{2}$ (soit un écart-type d'environ $8 \mathrm{~mm}$ ). Le modèle permet d'expliquer 93,3 p. 100 de la somme de carrés initiale avec ce seul axe. Il constitue donc de ce point de vue un «bon " résumé des variations de stock.

Les paramètres a (i) et $\mathrm{c}$ (i) de ce modèle sont maintenant étudiés plus précisément, dans la mesure où ils contiennent linformation spatiale que nous voulons privilégier.

- Les estimations des a (i) sont données dans la première colonne du tableau 3. Elles sont indépendantes les unes des autres et ont pour variance $66 / 94$ soit un écarttype d'environ $0,84 \mathrm{~mm}$.

- Celles des c (i) sont dans la deuxième colonne du tableau 3. Leur matrice de variance est $\left(I-C C^{\prime}\right) 3,5 / 1000$. Si ce résultat est appliqué au premier tube :

$\hat{c}(1)=0,026$ d'où une estimation de l'écart-type de $\sigma(\hat{c}(1))=0,006$

alors que $1 / \mathrm{t} \sqrt{23}=0,209$ serait la valeur théorique de $\mathrm{c}$ (i) si tous les tubes étaient identiques. Cette hypothèse est donc bien rejetée. 
TABLEAU 3

Modèle d'interaction : estimation des paramètres a et $c$ sur la parcelle feuillue.

Estimation of $a$ and $c$ parameters of the interaction model in the beech stand.

\begin{tabular}{c|c|c}
\hline Tube & $\hat{\mathbf{a}}(\mathrm{i})$ & $\hat{\mathbf{c}}$ (i) \\
\hline 1 & 534 & 0,026 \\
2 & 511 & 0,117 \\
3 & 484 & 0,251 \\
4 & 489 & 0,310 \\
5 & 495 & 0,206 \\
6 & 521 & 0,117 \\
7 & 543 & 0,043 \\
8 & 529 & 0,118 \\
9 & 512 & 0,178 \\
10 & 498 & 0,220 \\
11 & 472 & 0,353 \\
12 & 500 & 0,214 \\
13 & 485 & 0,289 \\
14 & 513 & 0,174 \\
15 & 506 & 0,185 \\
16 & 516 & 0,211 \\
17 & 501 & 0,219 \\
18 & 504 & 0,223 \\
19 & 495 & 0,261 \\
20 & 530 & 0,143 \\
21 & 523 & 0,112 \\
22 & 477 & 0,284 \\
23 & 516 & 0,178 \\
\hline
\end{tabular}

b) Analyse des paramètres spatiaux

- La liaison a (i) - c (i)

La simple observation de la figure 1 nous montre une liaison étroite entre les paramètres a (i) et $c(i)$ : plus la moyenne baisse et plus les tubes ont de fortes fluctuations. Ceci suggère comme modèle possible :

$y(i, j)=M+t . c(i) \cdot b(j)+e(i, j)$ où $M$ serait le stock maximum d'un point.

Ce modèle pose toutefois de nombreux problèmes d'estimation (DENIS, 1983), sans pour autant faire gagner un nombre important de paramètres.

- Cartographie

Si on examine les paramètres $\hat{c}$ (i), en fonction de leur position spatiale (fig. 2), on note un gradient très important. Il coïncide bien avec la partition faite par AUSSENAC \& Granier (1979) sur cette même parcelle en ne prenant en compte que les variations maximales de stocks, entre la réserve hydrique minimale atteinte en 1976, et la reconstitution de la réserve en hiver 1977. 


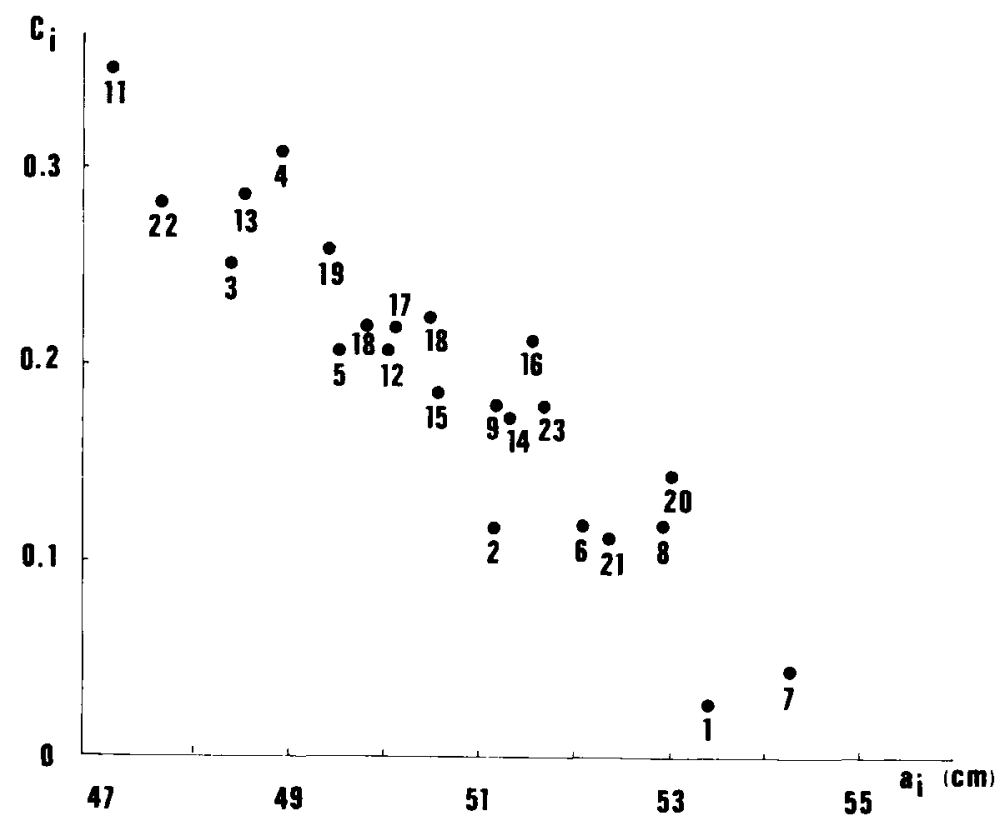

Fig. 1

Relation entre les coefficients a (i) et c (i) du modèle, pour les 23 tubes de la parcelle feuillue. Relationship between the a (i) and $c$ (i) parameters of the model for the 23 acces tubes of the beech stand.

\section{c) Conclusion}

Cette modélisation permet d'analyser les relations entre les variations de stock aux différents points de mesure. En particulier, les coefficients $\mathrm{c}$ (i), qui fournissent un résumé des proportions des variations de stock aux points mesurés peuvent être estimés avec d'autant plus de précision que l'on multipliera le nombre de dates de mesures. On a déjà pu observer ici une importante variabilité individuelle due en grande partie à un " gradient " d'humidité.

Afin de passer maintenant de la stricte observation à la prévision en d'autres points de la parcelle, il est nécessaire de modéliser ce gradient.

Au vu des cartes observées, nous avons choisi de le décrire comme une surface du second degré en fonction des deux coordonnées:

$c(i)=a x_{i}^{2}+b y_{i}^{2}+c x_{i} y_{i}+d x_{i}+e y_{i}+f+\epsilon_{i}$

où $\left(x_{i}, y_{i}\right)$ sont les coordonnées du point $i, \epsilon_{i}$ des variables aléatoires indépendantes gaussiennes de même variance. 


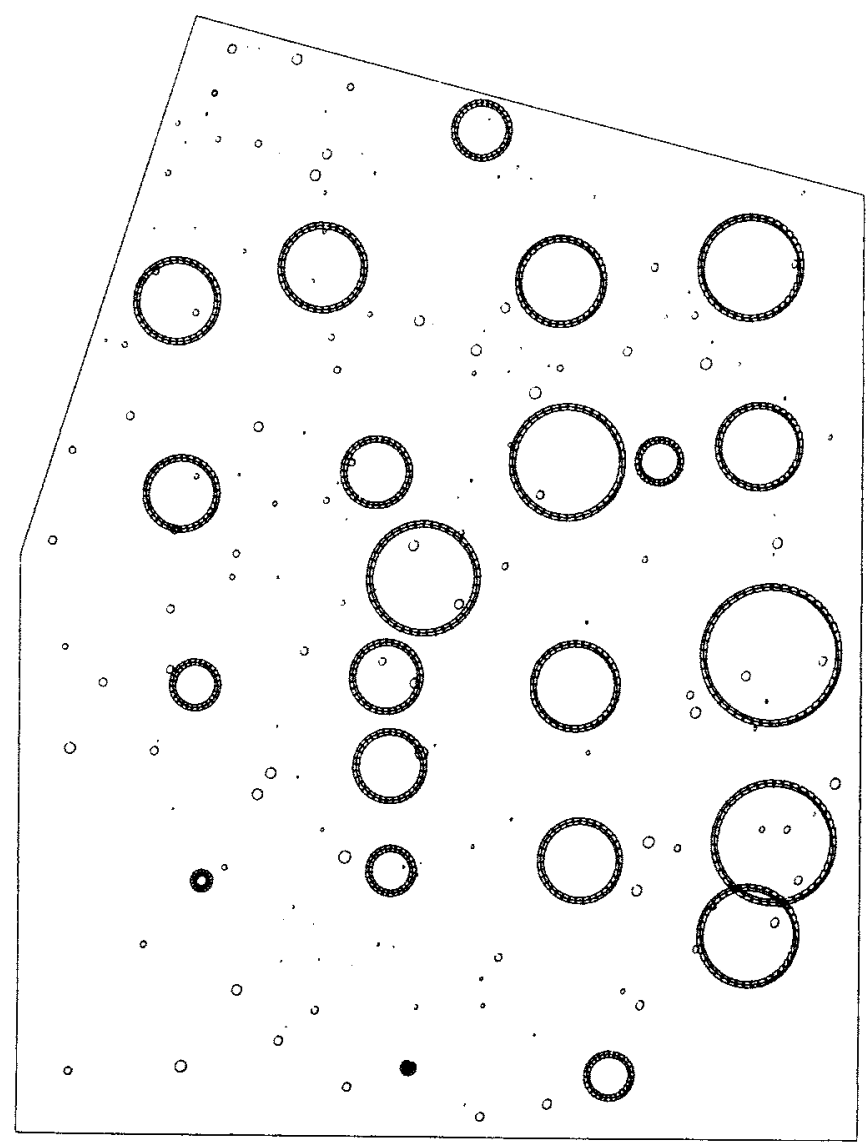

FIG. 2

Variation spatiale des paramètres c (i), reportée sur une carte de la parcelle feuillue. Le diamètre de chaque cercle est proportionnel à la valeur du coefficient $c(i)$, les cercles sont centrés sur les points de mesure.

Spatial variation of the $c$ (i) parameters for the beech stand. The diameter of each circle is proportional to the $c$ (i) value, and its center corresponds to the measurement point. 


\subsection{Modélisation des paramètres c (i)}

Si l'on considère alors un nouveau point $\mathrm{j}$ de la parcelle, on peut estimer la valeur c (j) du paramètre correspondant via la régression linéaire décrite ci-dessus. L'écart e (i) entre la vraie valeur et la valeur estimée traduit le biais systématique qui est fait sur l'évaluation des variations de stock en ce point.

\section{Estimation}

On obtient une bonne adéquation aux paramètres avec un coefficient de corrélation multiple de 0,85 et une erreur résiduelle de 0,05 . Les valeurs estimées ainsi que les résidus sont consignés dans le tableau 4.

\section{TABLEAU 4}

Estimation des coefficients $c$ (i) en fonction de leur position géographique, dans la parcelle feuillue, obtenus par régression linéaire du second degré des coordonnées $x$ et $y$ : $e$ (i) est l'écart entre la valeur réelle $c(i)$ et la valeur estimée $\hat{c}(i)$.

Estimation of the $c$ (i) coefficients as a function of their spatial coordinates in the beech stand; $e$ (i) is the difference between the actual value $c$ (i) and the estimated one $\hat{c}(i)$.

\begin{tabular}{c|r|r}
\hline \hline Tube & $\hat{\mathrm{c}}(\mathrm{i})$ & $\mathrm{e}(\mathrm{i})$ \\
\hline 1 & 0,024 & 0,002 \\
2 & 0,133 & $-0,016$ \\
3 & 0,261 & $-0,010$ \\
4 & 0,291 & 0,019 \\
5 & 0,202 & 0,004 \\
6 & 0,119 & $-0,002$ \\
7 & 0,042 & 0,001 \\
8 & 0,134 & $-0,016$ \\
9 & 0,184 & $-0,006$ \\
10 & 0,236 & $-0,016$ \\
11 & 0,298 & 0,055 \\
12 & 0,265 & $-0,051$ \\
13 & 0,237 & 0,052 \\
14 & 0,212 & $-0,038$ \\
15 & 0,189 & $-0,004$ \\
16 & 0,212 & $-0,001$ \\
17 & 0,204 & 0,015 \\
18 & 0,204 & 0,019 \\
19 & 0,205 & 0,056 \\
20 & 0,159 & 0,016 \\
21 & 0,251 & $-0,139$ \\
22 & 0,210 & 0,074 \\
23 & 0,159 & 0,019 \\
\hline \hline
\end{tabular}

On note un seul résidu particulièrement important. Il correspond au point 21 avec une valeur $\hat{\mathrm{c}}(21)=0,112$ pour une estimation par le modèle de 0,251 . Il s'agit là du 
seul effet des arbres dont nous avons pu démontrer l'existence. C'est en effet le seul point de mesure relativement éloigné des arbres : il est à 5 mètres de l'arbre le plus proche. De ce fait, le modèle surestime les variations de stock auxquelles ce point est soumis.

Si on reporte sur la carte l'effet ĉ estimé par le modèle précédent, nous obtenons la carte de la figure 3 . Cette carte ne contient pas en fait d'élément supplémentaire par rapport à ce que l'on pouvait déduire de la carte précédente. Signalons que l'on ne note pas de liaison, tant avec la pente qu'avec la proximité de la lisière. Elle met par contre plus en évidence la présence d'un point selle (point encadré par deux zones à fortes variations et deux à faible).

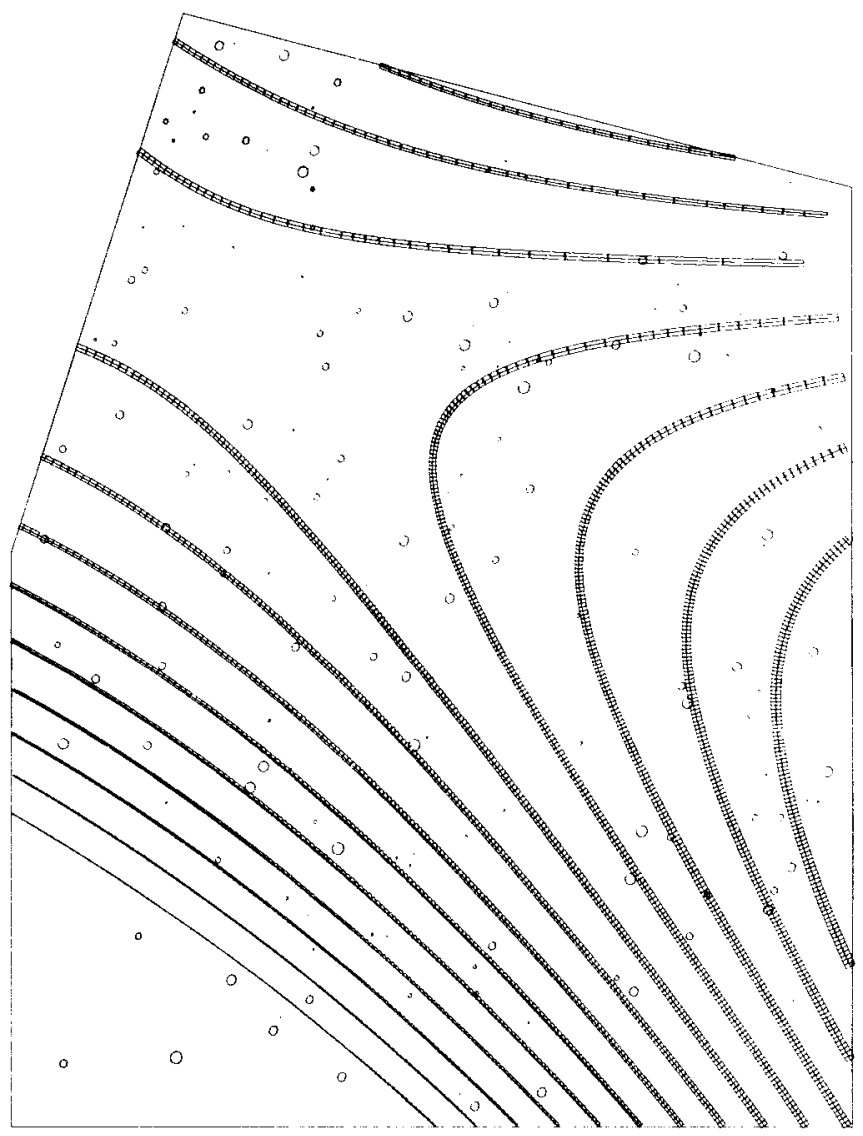

Fig. 3

Modélisation du gradient de l'effet c (voir texte) ; l'épaisseur de chaque courbe est proportionnelle $a \log (\hat{c})$.

Modelisation of the gradient of $c$ (i) (see text); the curves thickness is proportional to Log $(\hat{c})$. 
On retrouve bien les zones délimitées par Aussenac \& Granier, mais il y a en fait plus une variation continue, illustrée par la carte, qu'une séparation nette entre régions de comportements différents. L'échelle de variation est petite, puisque 9 mètres séparent par exemple les tubes 6 et 7 , qui fluctuent dans un rapport de 1 à 3 .

\subsection{Cas des peuplements résineux}

\subsection{Effet de la densité}

Une analyse similaire à celle décrite précédemment a été effectuée sur chacune des 3 parcelles du "carré latin" (cf. tabl. 1).

On obtient des ordres de grandeur des valeurs propres similaires à ceux observés dans la parcelle feuillue :

94,6 p. 100 d'inertie pour le premier axe, puis on tombe à 2,3 p. $100-0,8$ p. $100 \ldots$ c (i).

Le tableau 5 donne le premier vecteur propre ainsi que l'écart-type des paramètres

\section{TABLEAU 5}

Premier vecteur propre et écart-type de l'A.C.P. dans les trois plateaux de Douglas : DI : 535 tiges/ha, DII : 1030 tiges/ha, DIII : 2229 tiges/ha.

First eigen vector and standard deviation of the Principal Component Analysis in the three Douglas fir plots.

\begin{tabular}{|c|c|c|c|}
\hline & Tube & $1^{\text {er }} \mathrm{VP}$ & $\sigma$ \\
\hline DI & $\begin{array}{l}51 \\
52 \\
53 \\
54 \\
55 \\
56\end{array}$ & $\begin{array}{l}0,147 \\
0,156 \\
0,193 \\
0,122 \\
0,102 \\
0,140\end{array}$ & 0,036 \\
\hline DII & $\begin{array}{l}57 \\
58 \\
59 \\
60 \\
61 \\
62\end{array}$ & $\begin{array}{l}0,225 \\
0,239 \\
0,298 \\
0,262 \\
0,270 \\
0,245\end{array}$ & 0,035 \\
\hline DIII & $\begin{array}{l}63 \\
64 \\
65 \\
66 \\
67 \\
68\end{array}$ & $\begin{array}{l}0,308 \\
0,294 \\
0,256 \\
0,277 \\
0,312 \\
0,231\end{array}$ & 0,035 \\
\hline
\end{tabular}

On peut déjà noter un effet très important de la densité, dû principalement à l'écart entre la densité la plus faible et les deux autres parcelles. 
Une analyse de variance menée sur ces paramètres montre de ce fait un très important effet densité ( $f=32,0$ qui suit un $f(2,10)$, significatif au seuil de 5 p. 100), alors que l'effet position par rapport aux arbres n'est pas significatif.

\subsection{Effet de l'éclaircie}

Le modèle est appliqué année par année de façon à pouvoir suivre l'évolution des paramètres avec le temps.

Le tableau 6 donne l'évolution des trois premières valeurs propres avec le temps. On note par rapport aux exemples précédents une légère baisse de la première valeur propre les deux premières années après l'éclaircie (1980 et 1981).

\section{TABLEAU 6}

Evolution dans le temps du pourcentage d'inertie des trois premières valeurs propres de l'A.C.P. dans la parcelle de Douglas éclaircie.

Time evolution of the percentage of inertia of the three first eigen values of the Principal Component Analysis in the Douglas fir stand after thinning.

\begin{tabular}{cc|c|c|c}
\hline \hline \multicolumn{2}{c|}{ VP } & $1^{\text {er }}$ VP & $2^{\text {e VP }}$ & $3^{\text {e VP }}$ \\
\hline \multirow{2}{*}{1980} & 89,3 & 5,7 & 0,5 \\
1981 & 88,1 & 5,5 & 2,4 \\
1982 & 92,1 & 4,3 & 1,4 \\
1983 & 95,5 & 2,0 & 1,0 \\
1984 & 94,4 & 3,7 & 1,0 \\
\hline
\end{tabular}

Il y correspond principalement une augmentation de la seconde valeur propre. Cette dernière perd de son importance avec le temps et, dès les années 1983-1984, on retrouve des pourcentages d'inertie de 94 à 95 pour le premier axe. Cette évolution est plus nette si on étudie le premier vecteur propre (Cf. fig. 4) qui se montre plus sensible :

- on remarque l'évolution globale vers une stabilisation de l'état de la parcelle. Trois à quatre ans après l'éclaircie, la variabilité entre tubes est très faible, comparable à ce que l'on obtenait dans la parcelle du carré latin ;

— nous y retrouvons également « l'accident» de 1983 correspondant aux périodes où la parcelle avait été partiellement recouverte d'une bâche avec une augmentation de cette variabilité.

Toutefois, si on retrouve bien pour cet accident l'effet prévu (chute partielle de la variation des tubes 25-26-27-38-39 par rapport aux autres), l'effet dû à l'éclaircie est plus surprenant. On pouvait penser que les variations des stocks d'eau allaient être d'autant plus grandes que les tubes étaient proches d'arbres restés en place.

En fait, si cette logique est respectée pour les tubes 28-29-30-40-41, ce n'est plus vrai pour le deuxième groupe. En particulier, le tube 25 , le plus proche d'un arbre abattu lors de l'éclaircie, est celui qui fluctue le plus en 1980. 


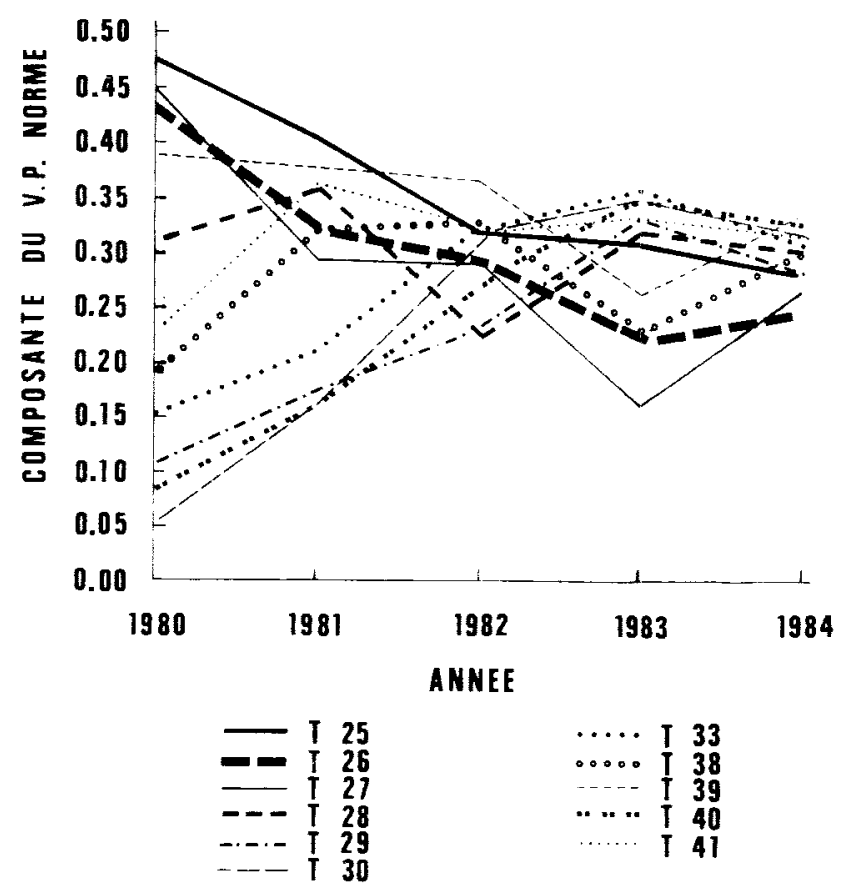

Fig. 4

Evolution sur les 5 années de mesures, du premier vecteur propre de l'A.C.P. effectuée année par année, pour la parcelle de Douglas éclaircie en 1980.

Evolution during the 5 studied years of the first cigen vector of the Principal Component Analysis, for the Douglas fir plot thinned in 1980 .

\section{Conclusion}

Le modèle statistique proposé a permis de décrire, au travers de ses paramètres, l'influence sur la distribution de l'eau dans le sol de phénomènes aussi différents que l'influence de l'éclaircie et son évolution au cours du temps, la variation de densité de plantation ou l'existence d'une nappe.

Il s'est montré bien adapté dans les périodes autres que les «accidents " (parcelles venant d'être éclaircies ou périodes de fortes sécheresses). Lorsque ces derniers arrivent, les paramètres décrivant la variation des stocks d'un point par rapport à un autre né peuvent plus être considérés comme constants (parce que le stock d'eau se vide peu à côté d'un arbre éclairci dans le premier cas, parce que le stock d'eau a atteint son niveau minimal en certains points dans le deuxième cas). Cest cette difficulté que nous 
avons partiellement contournée en traitant année par année les données issues des parcelles éclaircies, ces paramètres variant a priori relativement peu dans l'année, ce que confirme la bonne adéquation du modèle.

Cette étude est basée sur un nombre important de données, provenant surtout du grand nombre de dates de mesures. Par contre, les points de mesure sont en général beaucoup moins nombreux, nous conduisant à individualiser chacun d'eux dans l'analyse. Dans le cas le plus fourni, ces derniers nous ont permis de repérer et cartographier la nappe. La présence d'un plus grand nombre de points de sondage permettrait une étude spatiale plus fine au travers de méthodes statistiques plus appropriées telles que le krigeage, puis, une fois les aspects spatiaux analysés, de réduire l'échantillonnage à l'aide du modèle d'interaction proposé.

Reçu le 2 décembre 1987.

Accepté le 2 mai 1988.

\section{Références bibliographiques}

Aussenac G., Boulangeat C., 1980. Interception des précipitations et évapotranspiration réelle dans des peuplements feuillus (Fagus silvatica L.) et de résineux (Pseudotsuga menziesii (Mirb.) Franco). Ann. Sci. forest., 37 (2), 91-107.

Aussenac G., Granier A., 1979. Etude bioclimatique d'une futaie feuillue (Fagus silvatica L. et Quercus sessiliflora Salisb.) de l'Est de la France. II. Etude de l'humidité du sol et de l'évapotranspiration réelle. Ann. Sci. Forest., 36 (4), 265-280.

Aussenac G., Granier A., Naud R., 1982. Influence d'unc éclaircie sur la croissance et le bilan hydrique d'un jeune peuplement de Douglas (Pseudotsuga menziesii, Mirb. Franco). Can. J. For. Res., 12 (2), 222-231.

Chadoeuf J., 1984. Etude des transferts hydriques dans les peuplements forestiers. Analyse statistique des variations de stock et problème d'estimation liés à la technique du bilan hydrique. Thèse de $3^{\mathrm{e}}$ cycle, Université de Paris-Sud.

Denis J.B., 1983. Interaction entre deux facteurs. Thèse du Docteur Ingénieur. I.N.A.-P.G.

Milly P.C.D., Eagleson P.S., 1987. Effects of spatial variability on Annual Average Water Balance. Water Resour. Res., 23 (11), 2135-2143.

NiElsen D.R., Biggar J.W., ERH K.T., 1973. Spatial variability of field measured soil water properties. Hilgardia, 42 (7), 215-260.

Rambal S., Ibrahim M., RapP M., 1984. Variabilité spatiale des variations du stock d'eau du sol sous forêt. Catena, 11, 177-186.

Vauclin M., 1983. Méthodes d'étude de la variabilité spatiale des propriétés d'un sol. In "Variabilité spatiale des processus de transferts dans les sols ". Colloque INRA, n ${ }^{\circ} 15,1-43$. 